\title{
DEVELOPMENT OF A SILICON TWO-AXIS MICROMIRROR FOR AN OPTICAL CROSS-CONNECT
}

\author{
Andrew S. Dewa and John W. Orcutt \\ Telecommunications Business Unit, Materials \& Controls Group \\ Texas Instruments \\ Dallas, TX 75243 \\ Marshall Hudson and David Krozier \\ Telecommunications Business Unit, Materials \& Controls Group \\ Texas Instruments \\ Attleboro, MA 02703 \\ Alan Richards and Herzel Laor \\ Astarté Fiber Networks \\ Boulder, CO 80301
}

\begin{abstract}
This paper describes the development of a two-axis silicon micromirror for a $480 \times 480$ optical cross-connect (OXC). The micromirror is an electromagnetically actuated, analog silicon mirror. The range of motion for the micromirror is $\pm 8^{\circ}$. Full range of motion open-loop moves have been demonstrated in under $5 \mathrm{~ms}$, which enables a full $480 \times 480$ switch reconfiguration in $10 \mathrm{~ms}$. The micromirror and OXC have been optimized for low loss. The average optical insertion loss is $-2.5 \mathrm{db}$ at $1550 \mathrm{~nm}$ and $-2.9 \mathrm{db}$ at $1310 \mathrm{~nm}$, the two primary wavelengths in fiber optic data transmission. SONET data at $2.4 \mathrm{Gbits} / \mathrm{sec}$ has been transmitted through the OXC with no loss penalty.

The preliminary life and environmental validation data for the NxN Micromirror is reported. Bare micromirror samples have passed both the GR-63-Core vibration specification $(1-100 \mathrm{~Hz}$ 0.5G, 3-axis; $100-500 \mathrm{~Hz}, 3 \mathrm{G}, 3$-axis) and temperature extreme specification $\left(-40^{\circ} \mathrm{C}\right.$ to $\left.70^{\circ} \mathrm{C}\right)$ for devices in their shipping containers. We also have shock tested a small sample of micromirrors to failure. These fail at 800-900G shock. Micromirrors have been cycled to full deflection for 30 million cycles ( $6 \times$ specified life for the product) with no change in performance.
\end{abstract}

\section{INTRODUCTION}

The need for all-optical switching is being driven by exponentially increasing demand for bandwidth and the use of dense wavelength division multiplexing (DWDM) in all optical networks. As the number of wavelengths carried on each fiber grows, current methods of optical/electrical/optical (OEO) switching become unfeasible. In OEO switching, the light from one fiber must be first separated into its individual wavelengths. The time domain multiplexed signal on each wavelength is demulitplexed down to lower data rate electrical signals. The electrical signals are then switched. The signals are mulitplexed back up in data rate and the wavelengths are recombined for transmission to the output fiber. As the number of wavelengths per fiber grows, the space and power consumption of OEO quickly becomes too great.

In an all-optical cross connect (OXC), no electrical conversion is needed. All wavelengths are switched simultaneously. At present, all announced, large-port-count $(\mathrm{N} \geq 256)$ OXCs are MEMS-based, because of the ability of this technology to produce high-precision, micromechanical moving mirrors. Typical singlemode optical fiber cores are $9 \mu \mathrm{m}$ in diameter, so that the emerging optical beam is compatible with the size of typical MEMS optical components. The actual size of the micromechanical mirror depends on a complicated relationship of angle of view, the beam diameter, optical magnification, and the length of the optical path. Furthermore, since each mirror needs individual control and an optical fiber interface, the control electronics together with optical connector interfaces to support hundreds to thousands of micromirrors can dwarf the optical MEMS. In addition, the telecommunications industry requirements on system reliability mandate redundant power supplies and system controllers, so that the final size of the OXC system can be several equipment racks.

Texas Instruments (TI) and Astarté Fiber Networks (AFN) are jointly developing a $480 \times 480$ OXC. The TI/AFN approach utilizes individually-packaged, micromachined silicon mirrors to perform the switching function and active optical feedback to maintain low-loss, long-term connections. This approach provides a high degree of modularity, so that the customer can purchase the number of ports needed now, and further populate the switch frame as demand increases. Modularity also makes the OXC field serviceable-a failed module can be replaced without disrupting traffic on other modules.

This paper will discuss the development of the two-axis silicon micromachined mirror. It will include a general description of the OXC architecture, the fabrication and operation of the micromirror, preliminary life and environmental validation testing results, and the optical performance of the micromirror in the switch matrix. For clarity, the silicon mirror will be referred to as the NxN Micromirror throughout the rest of the paper.

\section{OPTICAL CROSS CONNECT}

Texas Instruments and Astarté Fiber Networks are jointly developing a $480 \times 480$ free-space, optical cross connect (OXC) $[1,2]$. The OXC is single-stage and non-blocking, allowing any-toany connectivity. TI is developing the Optical Unit (OU) capable of switching the optical signals from one fiber to another, while AFN has developed the overall OXC architecture. The NxN Micromirror provides the beam steering function for the OU. The OU also contains the focusing optics and the servo control emitters and detectors. AFN combines 4 OU's with a control electronics board into an Optical Module. The optical module is the minimum granularity of the OXC. Two hundred and forty modules, arranged in a 5 by 24 array make up a fully populated switch. All the optical units are pointed at a fixed mirror one meter away to give a 2-meter optical path length. A schematic showing four Optical Modules is given in Fig. 1.

The fundamental optical path of the OXC is shown in Fig 2. The light exits the fiber and goes through focusing optics. The expanded beam is reflected off a fixed mirror to the $\mathrm{NxN}$ Micromirror. The beam is steered by the $\mathrm{NxN}$ Micromirror to the 


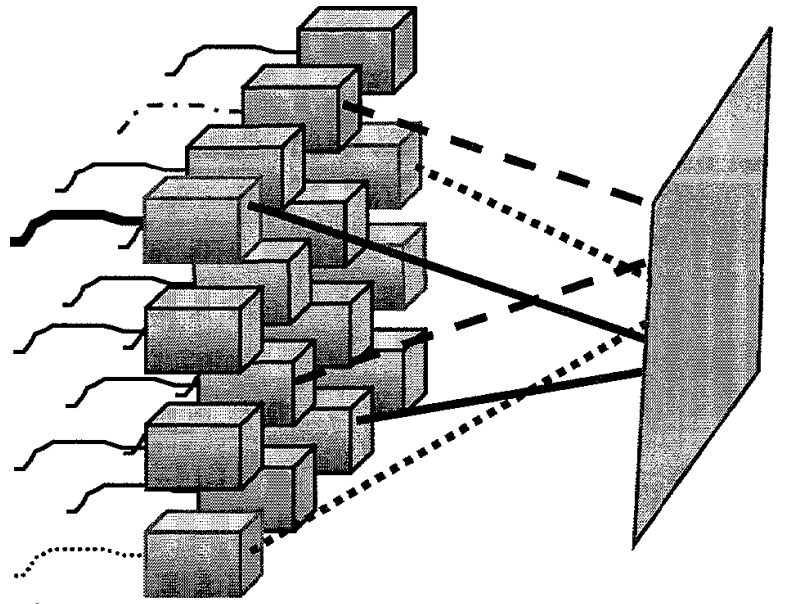

Figure 1. A schematic drawing showing 4 optical modules.

target OU via a large fixed mirror. This folding of the optical path allows for a smaller OXC footprint. It also allows for the doubling of the size of the switch, by replacing the mirror by another array of optical modules.

The two Micromirrors are servo controlled by emitters and detectors using the same optical path as the light from the fibers. Active servo control is key to achieving and maintaining very low insertion loss. The active servo control means that the connection is not susceptible to vibration, shock and long term drifts.

\section{NXN MICROMIRROR FABRICATION}

The NxN Micromirror is an electromagnetically-driven, analog, two-axis silicon mirror. A photograph of the mirror die is

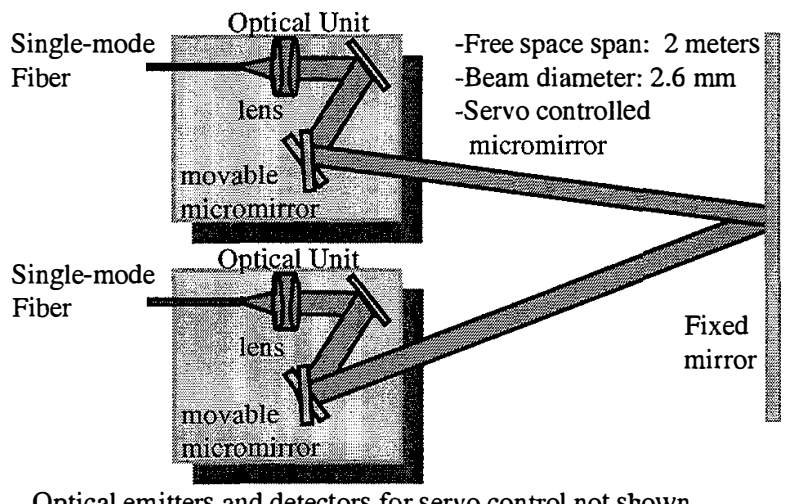

Optical emitters and detectors for servo control not shown

Figure 2. The optical path of the $480 \times 480$ OXC.

shown in Fig. 3. The active part of the silicon mirror is a $3.2 \times 3.6$ $\mathrm{mm}$ oval which is supported by 2 sets of silicon torsional hinges, one set between the mirror and the gimbals, and the second set between the gimbals and the frame. The size of the mirror is set by the beam diameter in the optical path. The two sets of torsional hinges allow the independent movement of the mirror in two axes. The overall die size is $7.2 \mathrm{~mm} \times 9.0 \mathrm{~mm}$. The mirror is about 0.1 $\mathrm{mm}$ thick and is gold coated to maximize its reflectivity at the wavelengths of interest for telecommunications, $1.25 \mu \mathrm{m}$ to 1.65 $\mu \mathrm{m}$.

The NxN Micromirror is fabricated using photolithography and reactive ion etching via the Bosch Process [1]. The etch recipe was optimized for vertical sidewalls with little undercut. The typical difference between the width of the torsional hinges at the

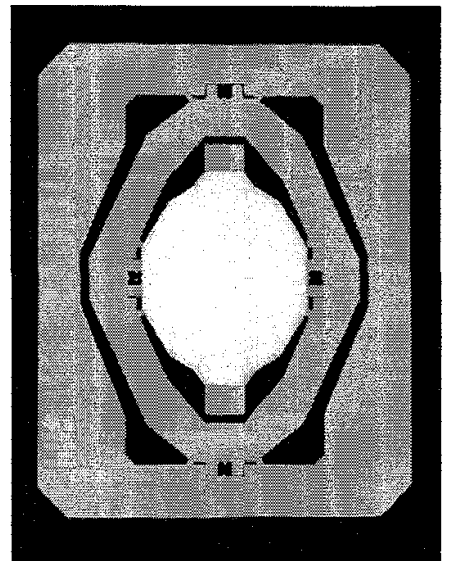

Figure 3. Optical photograph of an NxN Micromirror die.

top of the wafer and the bottom of the wafer is $0.2 \mu \mathrm{m}$ for the $100 \mu \mathrm{m}$ etch depth.

The packaged NxN Micromirror is shown in Fig. 4. The package was partitioned into a hermetic portion, the ceramic header and window that holds the NxN Micromirror, and a non-hermetic portion, where the coils for the actuator, wiring hamess and mounting bracket are located. The micromirror is hermetically packaged to guarantee the 20-year life expected by the

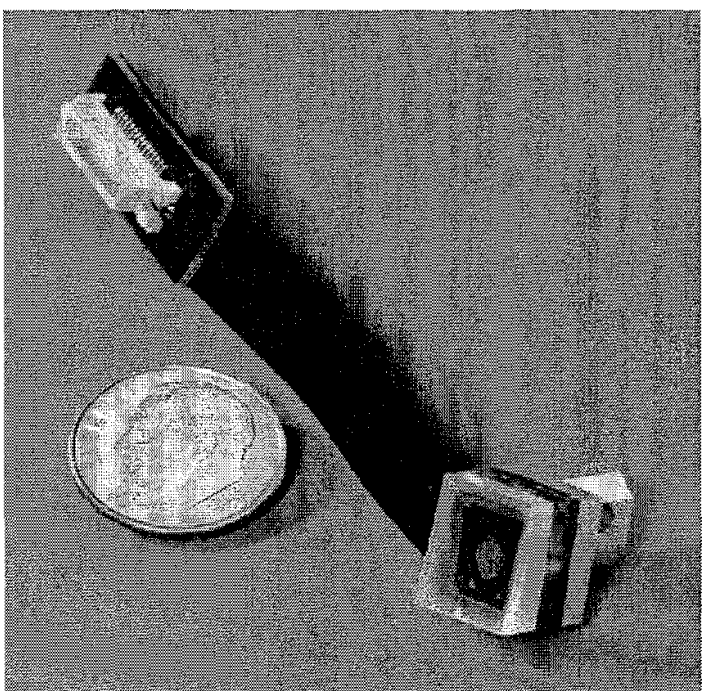

Figure 4. A photograph of the packaged NxN Micromirror. A US dime is shown for scale.

telecommunications industry.

\section{MICROMIRROR ACTUATOR}

The electromagnetic actuator for the mirror utilizes drive coils below the micromirror and permanent magnets mounted on the mirror and gimbals frame, as shown in Fig. 5. This actuator was chosen to allow the tips of the mirror and the gimbals to move several hundred microns providing the required deflection of $\pm 8^{\circ}$ in both axes with the millimeter-size of the micromirror.

Figure 6 shows actual deflection data from an $\mathrm{NxN}$ Micromirror prototype plotted on the magnetic model curves. Because there is no ferromagnetic material in the magnetic circuit other than the permanent magnets, analytical methods could be used to simulate the actuator [4]. This model, along with finite element analysis, was used to optimize the actuator design. The 


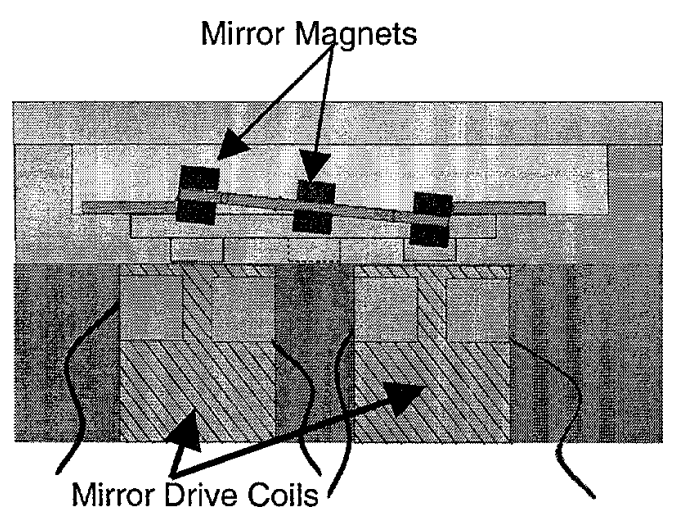

Figure 5. Schematic drawing of NxN Micromirror actuator mechanism.

mirror and gimbals hinges are identical in geometry and fall on the same straight line. Perfectly linear behavior is what is expected for single crystal silicon hinges. The model fits the experimental data to within the experimental errors of the measurement.

The OXC makes a connection by first steering the beam to the new location as fast as possible with open-loop control. Then an acquisition takes over to quickly move the remaining distance to the center position, at which point the closed-loop servo control takes over. Since the hinges are perfectly elastic, the micromirror is an underdamped system. Therefore, the dynamics of the system were modeled and a specified waveform is generated for each move. A move of $15.6^{\circ}$ of the mirror plus gimbals under openloop drive is shown in Fig 7.

The mirror plus gimbals moved 15.6 degrees in under $5 \mathrm{~ms}$ and was stable. The stability shown in Fig. 7 is more than enough for the acquisition and servo control to take over and optimize the connection. The under $5 \mathrm{~ms}$ move time is also sufficient to guarantee a full $\mathrm{OXC}$ reconfiguration in $10 \mathrm{~ms}$.

\section{ENVIRONMENTAL AND LIFE VALIDATON TESTING}

Key to deploying a product in the fiber optic telecommunication market is demonstrating a reliable product which meets the Telcordia specifications. We have begun validation tests on the environmental and life of the $\mathrm{NxN}$ Micromirror. As one would expect with single-crystal silicon hinges, cycling mirror over its full range of motion in both axes for over 30 million cycles results in no measurable change in the

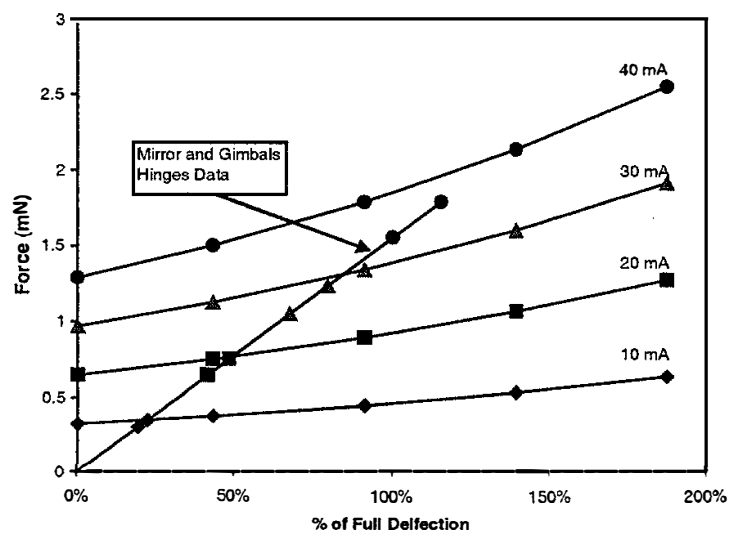

Figure 6. Measured hinge force vs. mirror deflection data plotted on the magnetic force model.

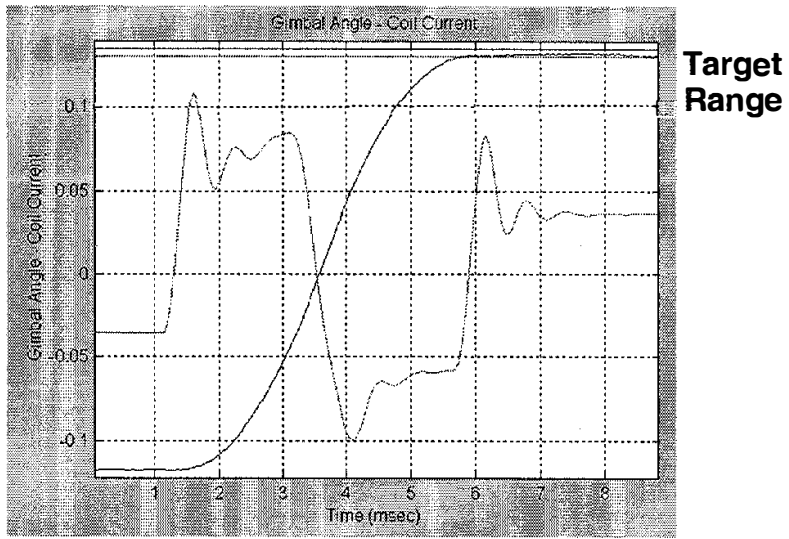

Figure 7. Measured position and current waveform for an open-loop move of $15.6^{\circ}$ in under $5 \mathrm{~ms}$ (Note: on the Y-axis the angle is in radians and the current is in amperes).

micromirror performance. Thirty million cycles is six times the product life specification of one reconnect every minute for 20 years. Since each cycle is move forward and back, one represents cycle two connections.

We have tested the NxN Micromirror to the GR-63-Core shipping temperature specification $-40^{\circ} \mathrm{C}$ to $+70^{\circ} \mathrm{C}$. The initial test samples have passed this test with no change in operating characteristics. Further testing is underway with additional samples to improve out confidence levels.

Upon seeing the $\mathrm{NxN}$ Micromirror, the initial reaction of most engineers is to question its shock and vibration sensitivity-because there are relatively large magnets at the ends of long moment arms supported by thin silicon hinges. To validate the design, we have begun testing the NxN Micromirror to the more severe GR-63Core specification for devices shipped in turboprop aircraft. It should be emphasized that the GR-63-CORE specification is for devices in their shipping containers, including all padding, etc. We tested 4 samples of the $\mathrm{NxN}$ Micromirror bolted directly to the shaker table to this specification: $1-100 \mathrm{~Hz}$ at $0.5 \mathrm{G}$ and $100-500 \mathrm{~Hz}$ at $3 \mathrm{G}$ in all three principal axes. (The standard GR-63-CORE shipping test is $100-500 \mathrm{~Hz}$ at $1.5 \mathrm{G}$.) All samples passed the test. Again, further testing is underway to improve our confidence levels.

We have also tested NxN Micromirrors to failure under shock. Three mirrors passed a 500G shock test with a 6 ms duration, in all three axes. We took 2 of the mirrors to failure under $800-900 \mathrm{G}$ shock tests with a duration of $2.5 \mathrm{~ms}$. The duration of the shock at higher $\mathrm{G}$ levels was reduced due to equipment limitations. Further shock testing is underway.

The environmental and life validation testing results to date are summarized in Table 1.

Table 1. Environmental and life test results

\begin{tabular}{|l|l|}
\hline Life Testing & 30 million cycles (3x expected life) \\
\hline $\begin{array}{l}\text { Shipping Thermal } \\
\text { Extremes }\end{array}$ & $\begin{array}{l}-40^{\circ} \mathrm{C} \text { to } 70 \mathrm{C} \text { with no detectable change } \\
\text { in performance }\end{array}$ \\
\hline Shock survivability & Survives 500G 3-axis, 6 ms duration \\
\hline Shock failure & $800-900 \mathrm{G} 2.5 \mathrm{~ms}$ duration \\
\hline $\begin{array}{l}\text { Vibration test } \\
\text { (GR-63-Core in } \\
\text { shipping container) }\end{array}$ & $\begin{array}{l}1-100 \mathrm{~Hz} 0.5 \mathrm{G}, 3 \text {-axis; } 100-500 \mathrm{~Hz}, 3 \mathrm{G}, \\
\text { 3-axis }\end{array}$ \\
\hline
\end{tabular}




\section{OPTICAL PERFORMANCE}

The OXC is designed to operate at the two major wavelengths utilized in optical fiber communications, $1310 \mathrm{~nm}$ and $1550 \mathrm{~nm}$. The coatings on the optical unit lens, optical elements and the micromirror window are optimized for the band-pass from 1250 to $1650 \mathrm{~nm}$ so that the OXC is fully compatible with dense wavelength division multiplexing.

The measured optical insertion loss at $1310 \mathrm{~nm}$ averaged -2.9 $\mathrm{db}$ with a maximum value of $-3.2 \mathrm{db}$. At $1550 \mathrm{~nm}$ the insertion loss averaged $-2.5 \mathrm{db}$ with a maximum value of $-3.0 \mathrm{db}$. Further testing of the optical loss is ongoing.

One key metric for an optical switch is to measure any excess data loss in a real telccommunications data channel other than that due to the loss of optical power measured by its insertion loss. This is done by comparing the data loss rate for the $\mathrm{OXC}$ to that of a passive optical attenuator with the same attenuation as the measured insertion loss of the connection. This is called the optical loss penalty. The OXC was able to transmit $2.4 \mathrm{Gbits} / \mathrm{sec}$. of SONET data with no measurable loss penalty.

The measured optical performance of the NxN Micromirror is

Table 2. NXN Micromirror OXC Performance

\begin{tabular}{|l|l|}
\hline $\begin{array}{l}\text { Optical Loss } \\
(1310 \mathrm{~nm})\end{array}$ & $-2.9 \mathrm{db}$ ave.; $-3.2 \mathrm{db}$ max. \\
\hline $\begin{array}{l}\text { Optical Loss } \\
(1550 \mathrm{~nm})\end{array}$ & $-2.5 \mathrm{db}$ ave.; $-3.0 \mathrm{db}$ max. \\
\hline $\begin{array}{l}\text { 2.4 Gbits/sec SONET } \\
\text { Data transmission }\end{array}$ & No Measurable Loss Penalty \\
\hline $\begin{array}{l}\text { Time for a full range } \\
\text { move }\end{array}$ & $5 \mathrm{~ms}$ for $15.6^{\circ}$ move (measured) \\
\hline $\begin{array}{l}\text { Time for a full switch } \\
\text { reconfiguration }\end{array}$ & $10 \mathrm{~ms}$ (projected) \\
\hline Deflection & $\pm 8^{\circ}$ in two independent axes \\
\hline
\end{tabular}

summarized in Table 2.

\section{SUMMARY}

We have demonstrated a two-axis silicon micromirror for a $480 \times 480$ optical cross connect. The micromirror is electromagnetically actuated, and can move, open-loop, $15.6^{\circ}$ in under $5 \mathrm{~ms}$ to enable a full switch reconfiguration in $10 \mathrm{~ms}$. The average optical insertion loss for the optical switch is $-2.5 \mathrm{db}$ at $1550 \mathrm{~nm}$ and $-2.9 \mathrm{db}$ at $1310 \mathrm{~nm}$. The optical switch has transmitted 2.4 Gbits/sec SONET data with no loss penalty.

We have begun life and environmental testing of the $\mathrm{NxN}$ Micromirror. Bare micromirror samples passed the GR-63-Core vibration specification and temperature extreme specifications for devices in shipping containers. We also shock tested a small sample of micromirrors to failure. These failed at $800-900 \mathrm{G}$. We tested micromirrors to 30 million cycles ( $3 \times$ expected life) with no change in performance. Further life and environmental testing is ongoing to get enough data to fully predict the life and reliability of the NxN micromirror.

\section{REFERENCES}

1. D. Krozier, M. Hudson, J. D'Entremont, H. Laor, A. Richards, and E. Fontenot, "Performance of a $576 \times 576$ Optical Cross Connect", Technical Proceedings of the National Fiber Optic Engineers Conference, Chicago, Illinois, September 26-30, 1999, vol. 1, p276.
2. H. Laor, A. Richards, E. Fontenot, "576x576 Optical Cross Connect for Single-Mode Fibers", Conference Proceedings of the 1999 Annual Multiplexed Telephony Conference, San Diego, CA, July 19-22, 1999, pp. 343-349.

3. F. Laermer and A. Schilp, Robert Bosch GmbH, "Method for Anisotropic Plasma Etching of Substrates", U.S. Patent 5498312 (1996).

4. E. P. Furlani, "A Three-dimensional Field Solution for Axially Polarized Multipole Disks," Journal of Magnetism and Magnetic Materials, 135, 205-214 (1994). 